culated based on the variation of the SFR shown in Figure 1 fill the region enclosed by the solid line in Figure 3 .

As for the spatial distribution of star forming regions, nuclear starbursts are often reported observationally. On the other hand, in our models the outer part of the disk is activated more efficiently (Figure 2). A more realistic simulation is needed to find out whether other factors neglected in our model are important in solving this discrepancy.

\title{
REFERENCES
}

Larson, R.B. and Tinsley, B.M.: 1978, Astrophys. J. 219, 46.

Noguchi, M. and Ishibashi, S.: 1986, Monthly Notices Roy. Astron. Soc. 218 (in press).

\section{CO OBSERVATIONS OF BRIGHT IRAS GALAXIES}

D.B. Sanders

Caltech, Pasadena, CA, USA

CO emission has been detected from 75 bright infrared galaxies with $\mathrm{CZ}=2000-16000 \mathrm{~km} / \mathrm{s}$. These include the most distant and the most luminous galaxies (Arp 55, IR 1713+63) yet detected in C0. All of these galaxies are rich in molecular gas with $\mathrm{M}_{\text {total }}\left(\mathrm{H}_{2}\right)=2 \times 10^{9}-$ $6 \times 10^{10} \mathrm{M}_{\odot}$, and they have a strong far-infrared excess, with $\mathrm{L}_{\mathrm{FIR}} / \mathrm{L}_{\mathrm{B}}=$ $2-40$ and $L_{F I R}(40-400 \mu)=10^{10}-3 \times 10^{12} L_{Q}$. The primary 1uminosity source appears to be star formation in molecular clouds. A strong correlation is found between the FIR and $21-\mathrm{cm}$ continuum flux, implying that the IMF is independent of the star formation rate. The ratio $\mathrm{L}_{\text {FIR }} /$ $\mathrm{M}\left(\mathrm{H}_{2}\right)$ provides a measure of the current rate of star-formation, which is found to be a factor 3-20 larger in these galaxies than for the ensemble of molecular clouds in the Milky Way. VLA maps plus a few high resolution (14"-30") CO (1-0) and CO (2-1) maps suggest that most of the luminosity comes from core regions $1-3 \mathrm{kpc}$ in size. The abnormal concentration of molecular gas in these galactic cores is presumably the result of a collision or strong interaction with a nearby companion. 